\title{
VARIACIÓN ESPACIAL Y TEMPORAL DEL FITOPLANCTON CON ÉNFASIS EN LAS FLORACIONES ALGALES FRENTE A LA PLAYA DE PESCADORES ARTESANALES DE CHORRILLOS / LIMA / PERÚ
}

\author{
SPATIAL AND TEMPORAL VARIATION OF PHYTOPLANKTON WITH \\ EMPHASIS IN ALGAL BLOOMS ON FISHERMEN CRAFTS IN FRONT OF A \\ BEACH AT CHORRILLOS / LIMA / PERU
}

\author{
Maribel Baylón ${ }^{1,2}$, Orlando Advíncula ${ }^{3}$, Omar Loyola $^{1,4}$, Ángel Norabuena ${ }^{1,5}$ y David Hernández-Becerril ${ }^{6}$
}

\section{Resumen}

Este estudio examinó la variabilidad espacial y temporal de las principales comunidades del fitoplancton en tres estaciones de la playa de Pescadores Artesanales de Chorrillos, Lima, Perú, en relación con las variables fisicoquímicas. Se realizaron mediciones in situ de temperatura y $\mathrm{pH}$ todos los meses durante un año y salinidad y oxígeno disuelto durante medio año. Se colectaron muestras de agua superficial para determinar la composición del fitoplancton. Se identificcaron un total de 120 taxa de organismos fitoplanctónicos, pertenecientes a 46 familias y 66 géneros. Las especies de fitoplancton fueron dominadas por las diatomeas (59.2\%) y dinoflagelados (35.6\%). La abundancia del fitoplancton estuvo dominada por Raphidophyceae (73.72\%), seguido de dinoflagelados $(11.75 \%)$ y nanoflagelados $(<20 \mu \mathrm{m})(11.53 \%)$. Asimismo, se registraron floraciones algales originadas por las especies, Thalassionema nitzschioides, Skeletonema costatum, Akashiwo sanguinea, Heterosigma akashiwo, Prorocentrum gracile, P. balticum, P. minimum, Scrippsiella acuminata, Dictyocha fibula, Leucocryptos marina y nanoflagelados $(<20 \mu \mathrm{m})$ durante todo el periodo de estudio. La mayor abundancia de fitoplancton se observó en verano y otoño. Se encontraron correlaciones significativas entre las comunidades del fitoplancton y las variables fisicoquímicas. Se determinó que los factores abióticos como: oxígeno disuelto, temperatura, salinidad y $\mathrm{pH}$ favorecieron la presencia de las floraciones algales. La existencia de especies de fitoplancton potencialmente tóxico/nocivo sugiere un monitoreo frecuente de las floraciones de algas nocivas (FAN) en la playa de Pescadores Artesanales de Chorrillos.

Palabras clave: variación temporal, fitoplancton, floraciones algales, Chorrillos.

\begin{abstract}
This study examined the spatial and temporal variability of the phytoplankton communities and their physicochemical variables in three stations at Pescadores Artesanales beach, located in Chorrillos (Lima, Peru). In situ measurements of temperature and $\mathrm{pH}$ were made every month for a year, while salinity and dissolved oxygen for half a year. Surface water samples were collected to determine the phytoplankton composition. A total of 120 phytoplanktonic taxa were identified, belonging to 46 families and 66 genera. Phytoplankton species were dominated by diatoms $(59.2 \%)$ and dinoflagellates $(35.6 \%)$. Phytoplankton abundance was dominated by the Raphidophyceae $(73.72 \%)$ followed by dinoflagellates $(11.75 \%)$, and nanoflagellates $(11.53 \%)$. Algal blooms were formed by Thalassionema nitzschioides, Skeletonema costatum, Akashiwo sanguinea, Heterosigma akashiwo, Prorocentrum gracile, P. balticum, P. minimum, Scrippsiella acuminata, Dictyocha fibula, Leucocryptos marina and some nanoflagellates $(<20 \mu \mathrm{m})$. The highest phytoplankton abundance was observed in summer and autumn. Significant correlations were found between phytoplankton communities and physicochemical variables. Abiotic factors such as dissolved oxygen, temperature, salinity and $\mathrm{pH}$ favored the presence of algal blooms. Existence of potentially toxic / harmful phytoplankton species suggests a higher monitoring frequency of harmful algal blooms (FAN) at Pescadores Artesanales beach.
\end{abstract}

Key words: temporal variability, phytoplankton, algal blooms, Chorrillos.

\section{Introducción}

La costa de la ciudad de Lima se encuentra incluida en la Provincia de Lima, está caracterizada por sus aguas templadas, la cual está influenciada por los vientos alisios en dirección Sureste, que causan los afloramientos (Kameya, 2002), también llamados 
surgencias, siendo Paita, Punta Aguja, Chimbote, Callao y San Juan las principales zonas de afloramientos. Estas surgencias resultan en una alta disponibilidad de nutrientes en la zona costera de Perú durante todo el año, la cual influye en las comunidades biológicas, en particular, las fitoplanctónicas (Graco et al., 2007).

La playa de Pescadores Artesanales de Chorrillos es uno de los lugares más importantes y turísticos del circuito de playas de la Costa Verde del sur de Lima, cuenta con una amplia caleta de pescadores, quienes se encargan de extraer una variada población marina representada por 23 especies de peces y 9 de mariscos, con mayor ponderación estacional del Sarda sarda y la Sciaena deliciosa que en conjunto cubren el $70.2 \%$ del total de desembarques (Bustamante \& Borda, 1970).

$\mathrm{El}$ fitoplancton es la base de la cadena trófica, y su alta producción en áreas costeras peruanas sostiene la actividad económica de peces y mariscos, por ende, los estudios sobre las características y dinámicas de la comunidad fitoplanctónica permite conocer mejor el funcionamiento del ecosistema y de sus recursos (Ochoa \& Gómez, 1988). El fitoplancton marino destaca por su riqueza y abundancia de Bacillariophyta y Dinophyta. También sobresalen los fitoflagelados, organismos unicelulares micro-planctónicos $(<200$ $\mu \mathrm{m})$, nano-planctónicos $(<20 \mu \mathrm{m})$ e incluso ultraplanctónicos $(<2 \mu \mathrm{m})$ que destacan en la producción primaria, en la biomasa total y en la concentración total de clorofilas en el océano (Malone 1971a b; Throndsen, 1979; Hallegraeff, 1981, 1983; Booth et al., 1982; Hallegraeff \& Jeffrey, 1984; Hernández, 1993).

La composición y abundancia del fitoplancton son consideradas como bio-indicadores naturales de las variaciones en la calidad del agua debido a su sensibilidad y rápidas respuestas a los cambios de las condiciones ambientales como $\mathrm{pH}$, luz, temperatura, salinidad, turbiedad y nutrientes (Ekwu \& Sikoki 2006). Así, la composición de especies, abundancia relativa, distribución espacial y temporal de estas biotas acuáticas son una expresión de la salud ambiental o integridad biológica de un cuerpo de agua particular (Limbu \& Kyewalyanga, 2015).

Las floraciones algales (FA), por mucho tiempo llamadas "mareas rojas", han sido descritas a nivel mundial, caracterizándose por coloraciones anómalas del agua de mar ocasionadas por el elevado crecimiento de organismos fitoplanctónicos, mayormente dinoflagelados en concentraciones de $10^{6} \mathrm{cel} \cdot \mathrm{l}^{-1}$. Son las FA inocuas, inofensivas, en la mayoría de los casos; no producen toxinas, pero por las tonalidades que presentan pueden crear alarma innecesaria. En el caso de las floraciones algales nocivas (FAN) estas son ocasionadas por especies de microalgas (Dictyocha y Chaetoceros) cuya morfología puede causar algún daño mecánico a peces, como la obstrucción de sus branquias y ocasionar su muerte y finalmente las FA tóxicas son coloraciones anómalas productoras de potentes venenos que causan mortandad en peces y otros organismos marinos (Reguera, 2002).

En el Perú las FA han sido conocidas desde décadas pasadas como fenómenos naturales que producen coloraciones anómalas en el mar, precedidas por emanaciones de gas sulfhídrico, asociados a muerte de peces o aves guaneras. Hasta el momento se ha logrado identificar a los organismos productores de estos fenómenos estimándose sus concentraciones celulares (Sánchez \& Delgado, 1996).

Los estudios en floraciones algales en el litoral peruano han sido realizados por Rojas de Mendiola (1979), Santander \& Ochoa (1981), Sánchez \& Delgado (1996), Sánchez et al. (2015), Baylón et al. (2015) y Tillmann et al. (2017). Las investigaciones en floraciones algales inocuas y nocivas, se ubican

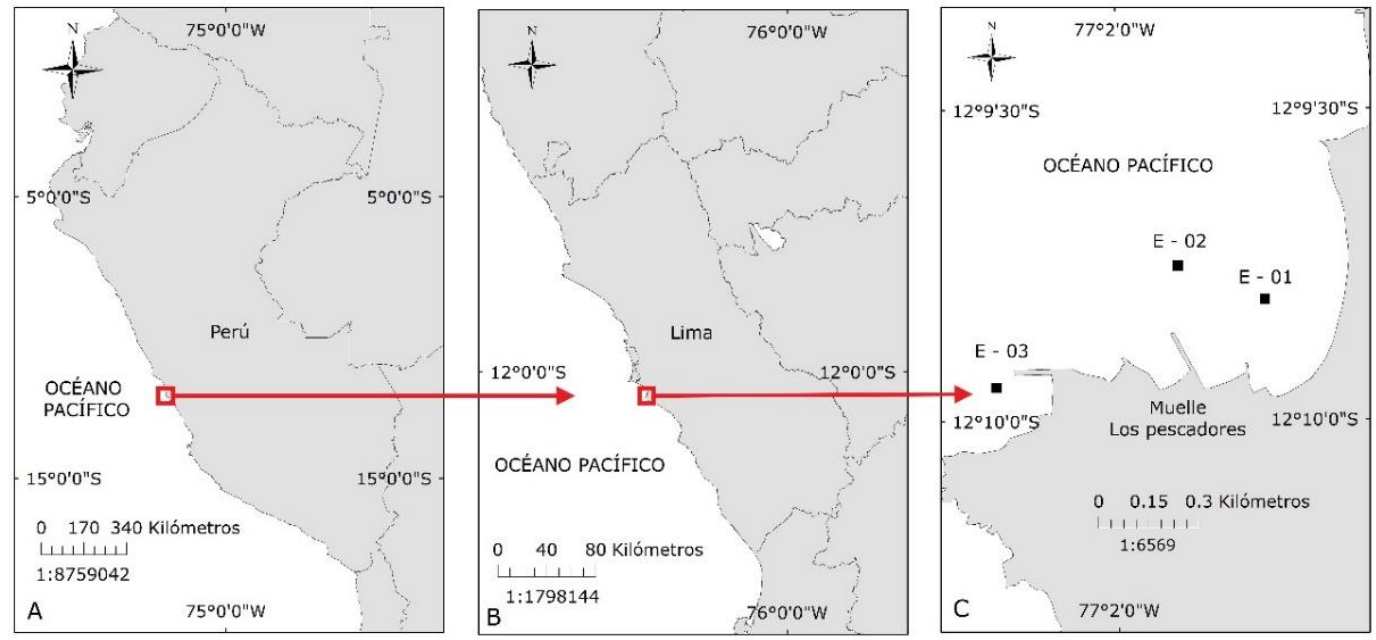

Figura 1. Mapa de la playa de Pescadores Artesanales de Chorrillos-Lima-Perú. A: Perú, B: Lima, C: Mapa de ubicación de las estaciones de muestreo en la playa de Pescadores Artesanales de Chorrillos. 
principalmente en áreas de cultivo de moluscos bivalvos como en Paita, Chimbote, Callao y Pisco. Este tipo de investigación es aún muy incipiente en los terminales pesqueros artesanales.

El objetivo del presente estudio fue determinar la variabilidad espacial y temporal de las principales comunidades del fitoplancton en tres estaciones de muestreo de la playa de Pescadores Artesanales de Chorrillos, Lima, Perú, en relación con las variables fisicoquímicas a lo largo de un año de estudio.

\section{Materiales y métodos}

Área de Estudio

La playa de Pescadores Artesanales de Chorrillos se encuentra en el distrito de Chorrillos en la provincia y departamento de Lima entre las coordenadas $12^{\circ} 09^{\prime} \mathrm{S}$ $12^{\circ} 10^{\prime} 0.4^{\prime \prime} \mathrm{S}$ y $77^{\circ} 01^{\prime}-77^{\circ} 02^{\prime} 14.4^{\prime \prime} \mathrm{W}$, estableciéndose tres estaciones de muestreo (E-01, E-02 y E-03) en la zona marina costera (Figura 1).

Muestreo de fitoplancton

Las muestras de agua y red se colectaron mensualmente, entre abril 2016 hasta marzo 2017. Las muestras cualitativas se colectaron con una red estándar de fitoplancton con abertura de malla de $10 \mu \mathrm{m}$, operada a través de arrastres horizontales a una velocidad de 3 nudos y tiempo de $5 \mathrm{~min}$, fijando el material con formalina neutralizada (4\%). Las muestras de agua para el análisis cuantitativo se colectaron con un recipiente a nivel superficial y preservadas con solución de lugol ácido (Throndsen, 1978). Durante los muestreos en que se evidenció coloraciones anómalas del agua de mar, se colectaron muestras in vivo para identificar dinoflagelados desnudos y nanoflagelados $(<20 \mu \mathrm{m})$.

\section{Variables fisicoquímicas}

Desde abril 2016 hasta marzo 2017, se evaluaron mensualmente los parámetros fisicoquímicos: la temperatura del agua y potencial hidrogeniones $(\mathrm{pH})$ se midieron con un multiparámetro marca HACH (modelo HQ11d) y la salinidad con un conductímetro marca HACH (modelo HQ14d) el cual fue previamente calibrado. El oxígeno disuelto se tomó con el oxímetro marca American Marine Inc (modelo PinPoint II). La toma de muestras de agua para determinar oxígeno y salinidad solo se evaluó entre octubre 2016 hasta marzo 2017, debido a dificultades en la adquisición de equipos.

\section{Procesamiento de muestras}

Para el análisis cualitativo se empleó un microscopio óptico Leica ${ }^{\circledR}$ con cámara ICC50 y se realizó un listado taxonómico considerando la presencia/ausencia de las especies. La determinación de las especies del fitoplancton se efectuó consultando los trabajos de Round et al. (1990), Hustedt (1930), Cupp (1943), Hasle \& Syvertsen (1997) para Bacillariophyta, Balech (1988), Balech (1995) y Licea et al. (1995) para Dinophyta y Throndsen (1993) para Raphidophyceae.

El análisis cuantitativo se realizó por el método de Utermöhl (1958) usando un microscopio invertido Leica y cubetas de sedimentación de $25 \mathrm{ml}$. Los resultados se expresaron en cel $\cdot \mathrm{l}^{-1}$. Las especies típicas, son aquellas que resultaron con valores de frecuencia de aparición de $50 \%$ considerando el número total de muestras revisadas $(\mathrm{n}=36)$.

Análisis de datos

Se realizaron gráficos para analizar la variación de la temperatura, $\mathrm{pH}$, salinidad y oxígeno respecto al tiempo y las 3 estaciones de muestreo. Para analizar si hubo variación significativa respecto a las estaciones de muestreo, se usó el análisis de varianza para cada una de las 4 variables físico-químicas. Se verificó el cumplimiento de los supuestos. Para analizar la variación de Bacillariophyta (diatomeas), Miozoa (dinoflagelados), Raphidophyceae y nanoflagelados (< $20 \mu \mathrm{m})$, respecto a las estaciones de muestreo, se usaron modelos lineales generalizados con la familia Binomial Negativa, por tratarse de variables de conteo.

Debido a que no se encontraron diferencias significativas entre las variables fisicoquímicas respecto a los puntos de muestreo (Anova, $\mathrm{p}>0.05$ ); se realizó un análisis de varianza de estas variables respecto a los meses (se tomaron las 3 estaciones de muestreo como repeticiones); en casos que hubo diferencias se aplicó la prueba de comparaciones LSD de Fisher.

Para analizar la correlación entre las variables fisicoquímicas y las abundancias de Miozoa, Raphidophyceae, Euglenophyta, y nanoflagelados (< $20 \mu \mathrm{m})$ se usó la correlación de Pearson.

\section{Resultados \\ Variables fisicoquímicas}

\section{Temperatura}

Se observó que la temperatura osciló entre los 16 y $23.8{ }^{\circ} \mathrm{C}$, encontrando los valores más bajos entre los meses de mayo a agosto de 2016 y los valores más altos en el verano de 2017 (Tabla 1).

\section{pH}

Durante el periodo de estudio el pH osciló entre 6.69 y 8.32, evidenciándose los valores más bajos entre mayo a setiembre de 2016 y los más altos en el verano de 2017 (Tabla 1).

\section{Salinidad y oxígeno disuelto}

La salinidad osciló entre 34.3 y 35.9 \% y el oxígeno osciló entre 3.1 y $10 \mathrm{mg} \cdot \mathrm{l}^{-1}$ (Tabla 1). Los mayores valores de oxígeno y $\mathrm{pH}$ se registraron en la primavera 2016 (diciembre) y verano 2017 (marzo).

Composición de la comunidad fitoplanctónica.

Se identificó un total de 120 taxa de organismos fitoplanctónicos pertenecientes a 6 divisiones, 10 clases, 33 órdenes, 46 familias y 66 géneros, en las tres estaciones de muestreo durante los doce meses de 
Tabla 1. Variables fisicoquímicas en la playa de Pescadores Artesanales de Chorrillos, Lima, Perú, durante el periodo de estudio.

\begin{tabular}{|c|c|c|c|c|c|c|c|c|c|c|c|c|c|}
\hline 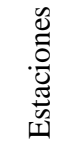 & Fecha & $\begin{array}{l}0 \\
\stackrel{1}{*} \\
\frac{\pi}{\sigma}\end{array}$ & $\begin{array}{l}0 \\
\stackrel{0}{1} \\
\stackrel{\Xi}{\Xi}\end{array}$ & $\begin{array}{l}\stackrel{0}{1} \\
\stackrel{1}{\Xi} \\
.\end{array}$ & $\frac{0}{\frac{1}{1}}$ & $\begin{array}{l}0 \\
0 \\
\vdots \\
0 \\
0 \\
0\end{array}$ & $\begin{array}{l}0 \\
\frac{1}{d} \\
\infty\end{array}$ & $\frac{0}{\frac{1}{d}}$ & $\begin{array}{l}0 \\
\frac{1}{1} \\
0 \\
0\end{array}$ & $\frac{0}{\frac{0}{\prime}}$ & $\frac{\bar{n}}{\dot{d}}$ & $\begin{array}{l}\frac{1}{1} \\
\stackrel{0}{0}\end{array}$ & 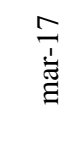 \\
\hline \multirow[t]{4}{*}{ E-01 } & $\begin{array}{l}\text { Temperatura } \\
\left({ }^{\circ} \mathrm{C}\right)\end{array}$ & 22.2 & 18.1 & 17.7 & 17.5 & 16.9 & 16.5 & 19 & 16.3 & 22 & 19 & 22.6 & 23.8 \\
\hline & $\mathrm{pH}$ & 7.8 & 7.06 & 6.69 & 6.79 & 7.02 & 7.05 & 7.7 & 7.64 & 8.31 & 7.89 & 7.98 & 8.32 \\
\hline & $\begin{array}{l}\text { Salinidad } \\
(\% \text { ) }\end{array}$ & & & & & & & 34.9 & 34.8 & 35.1 & 35.1 & 35.2 & 35.9 \\
\hline & $\begin{array}{l}\text { Oxígeno } \\
(\mathrm{mg} \cdot 1-1)\end{array}$ & 6.9 & & & & & & 4.4 & 5.8 & 9.1 & 4.7 & 3.2 & 10 \\
\hline \multirow[t]{4}{*}{ E-02 } & $\begin{array}{l}\text { Temperatura } \\
\left({ }^{\circ} \mathrm{C}\right)\end{array}$ & 22.1 & 17.8 & 17.6 & 18.7 & 16.8 & 18.7 & 19.1 & 16.1 & 22.2 & 19.9 & 22.6 & 23.5 \\
\hline & $\mathrm{pH}$ & 7.7 & 6.75 & 6.69 & 6.85 & 7.07 & 6.85 & 7.68 & 7.72 & 8.3 & 7.88 & 7.93 & 8.12 \\
\hline & $\begin{array}{l}\text { Salinidad } \\
(\% \text { ) }\end{array}$ & & & & & & & 35 & 34.9 & 34.9 & 34.8 & 34.9 & 35.6 \\
\hline & $\begin{array}{l}\text { Oxígeno } \\
(\mathrm{mg} \cdot 1-1)\end{array}$ & 7.6 & & & & & & 4.7 & 3.4 & 10.8 & 4.9 & 3.1 & 8.7 \\
\hline \multirow[t]{4}{*}{ E-03 } & $\begin{array}{l}\text { Temperatura } \\
\left({ }^{\circ} \mathrm{C}\right)\end{array}$ & 21 & 18.1 & 17.7 & 18.6 & 16.8 & 18.6 & 19.3 & 16 & 21.9 & 20.6 & 22 & 23 \\
\hline & $\mathrm{pH}$ & 7.69 & 6.77 & 6.75 & 7.07 & 7.06 & 7.07 & 7.6 & 7.63 & 7.27 & 7.6 & 7.69 & 7.87 \\
\hline & $\begin{array}{l}\text { Salinidad } \\
(\% \text { ) }\end{array}$ & & & & & & & 34.9 & 34.4 & 34.9 & 34.3 & 34.9 & 35.5 \\
\hline & $\begin{array}{l}\text { Oxígeno } \\
(\mathrm{mg} \cdot 1-1)\end{array}$ & 7.2 & & & & & & 5.3 & 3.6 & 8 & 5.8 & 3.8 & 7.5 \\
\hline
\end{tabular}

estudio. Las diatomeas fueron las más abundantes con 71 especies que representan el $59.2 \%$, seguido de los dinoflagelados con 43 especies representando el $35.6 \%$ (Figura 2).

Los géneros con mayor número de taxa fueron: Chaetoceros (12), Thalassiosira (6), Coscinodiscus (5), Protoperidinium (13), Tripos (5), Dinophysis (5) y Prorocentrum (4). Las especies con frecuencias mayores de 50\% corresponden a 14 diatomeas: Actinocyclus sp., Actinoptychus splendens, Amphora sp., Coscinodiscus perforatus, Coscinodiscus radiatus, Cyclotella sp., Navicula sp.1, Cylindrotheca closterium, Pleurosigma sp., Skeletonema costatum, Thalassionema nitzschioides, Thalassiosira angulata, Thalassiosira punctigera y Thalassiosira sp., un dinoflagelado: Prorocentrum gracile, dos silicoflagelados: Dictyocha fibula, Octactis octonaria y nanoflagelados $(<20 \mu \mathrm{m})$.

Variación espacial

Temperatura, $\mathrm{pH}$, salinidad y oxígeno no difieren significativamente entre las tres estaciones de muestreo (temperatura, $\mathrm{F}=0.04, \mathrm{gl}=2, \mathrm{p}=0.9580 ; \mathrm{pH}, \mathrm{F}=0.38$ $\mathrm{gl}=2, \mathrm{p}=0.6882 ;$ salinidad, $\mathrm{F}=1.32, \mathrm{gl}=2, \mathrm{p}=0.2977$ y oxígeno disuelto, $\mathrm{F}=0.06, \mathrm{gl}=2, \mathrm{p}=0.9398$ ). En estos 4 casos se cumplieron los supuestos de homogeneidad de varianzas y la distribución normal de los residuales.

Diatomeas, dinoflagelados, Raphidophyceae y

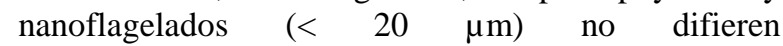
significativamente entre las tres estaciones de muestreo (diatomeas, $\mathrm{F}=1.06, \mathrm{gl}=2, \mathrm{p}=0.3594$; dinoflagelados, $\mathrm{F}=0.63, \mathrm{gl}=2, \mathrm{p}=0.5412$; Raphidophyceae, $\mathrm{F}=0.73$, $\mathrm{gl}=2, \mathrm{p}=0.4893$ y nanoflagelados $(<20 \mu \mathrm{m}), \mathrm{F}=$ 3.390, $\mathrm{gl}=2, \mathrm{p}=0.0458)$. Para todos los casos se comprobó que el cociente Deviance/ $\mathrm{N}$ fuera menor a 3 , lo cual indica un buen ajuste del modelo.

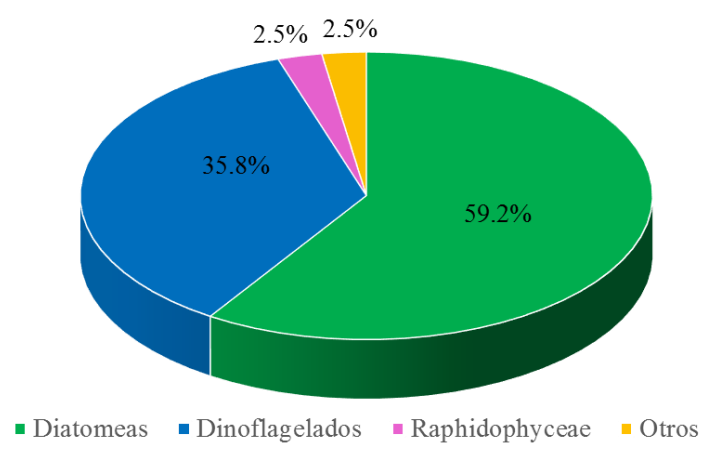

Figura 2. Composicion del fitoplancton en las tres estaciones de estudio en la playa de Pescadores Artesanales de Chorrillos, Lima, Perú.

Al no encontrar variaciones espaciales en las variables fisicoquímicas $y$ en las comunidades fitoplanctónica entre las tres estaciones de muestreo se procedió a examinar las variaciones temporales tomando a las estaciones de muestreo como repeticiones. 


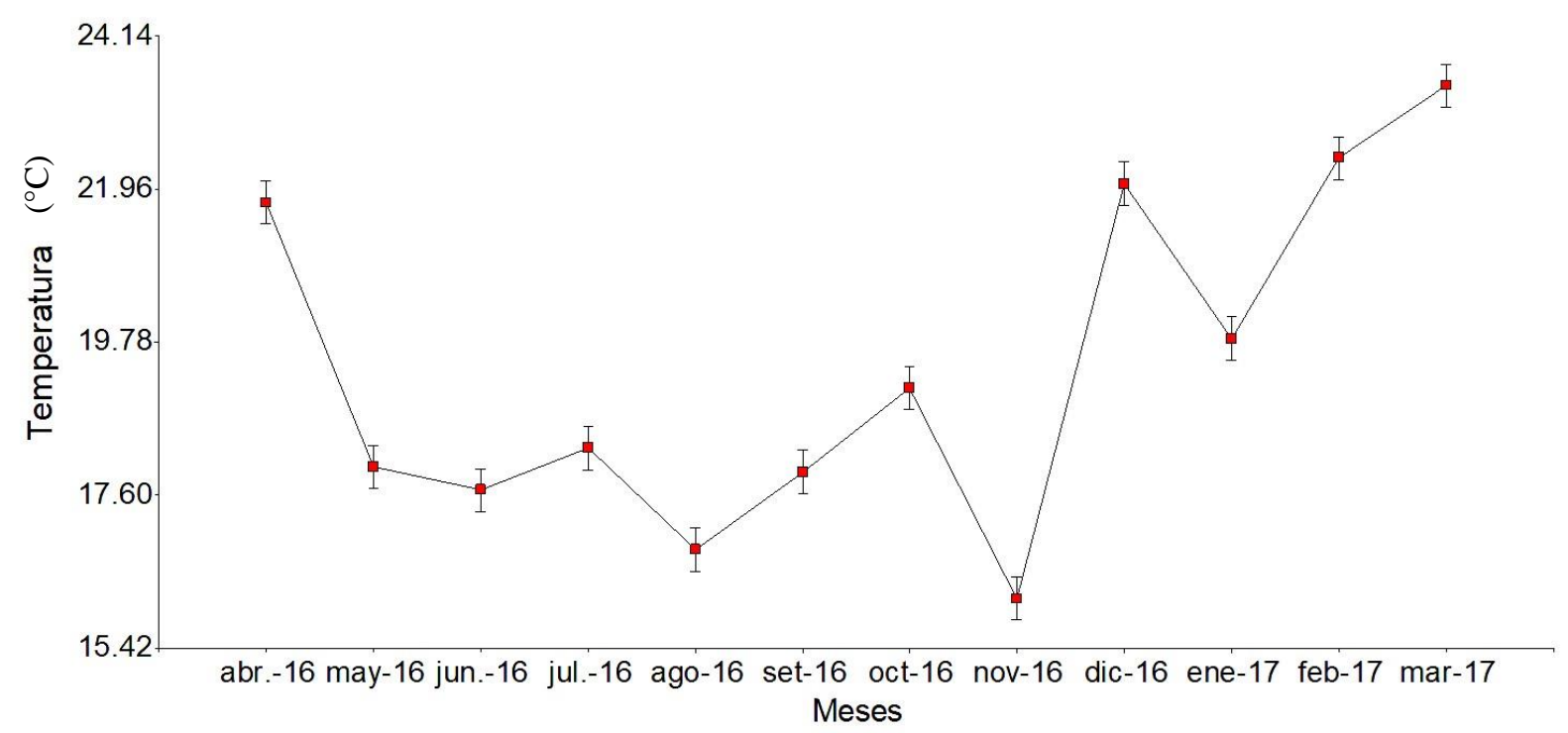

Figura 3. Variación temporal en valores medios $( \pm \mathrm{EE})$ de la temperatura del agua durante el periodo de estudio.

Variación temporal

\section{Variables fisicoquímicas}

La temperatura promedio más alta se registró en marzo de $2017\left(23.43 \pm 0.31{ }^{\circ} \mathrm{C}\right)$ y la más baja, en noviembre de $2016\left(16.13 \pm 0.31{ }^{\circ} \mathrm{C}\right)$. La temperatura del agua mostró diferencias significativas $(\mathrm{p}<0.05)$. En las comparaciones con el LSD de Fisher puede observarse que no hay diferencias significativas entre los meses abril 2016, diciembre 2016 y febrero 2017 y, así mismo, para los meses de junio, julio y septiembre de 2016 (Figura 3).

La salinidad promedio fue significativamente diferente entre los meses de muestreo $(\mathrm{F}=6.80, \mathrm{gl}=5$, $\mathrm{p}=0.0032$ ), encontrándose la salinidad más alta en marzo de $2019\left(35.67 \pm 0.13{ }^{\circ} \mathrm{C}\right)$. Para la salinidad, se encontraron diferencias significativas $(\mathrm{p}<0.05)$. En las comparaciones entre meses con el LSD de Fisher, indicó que no hay diferencias entre los meses de octubre del 2016 a febrero del 2017, pero si en marzo del 2017 donde la salinidad tomó su valor más elevado (Figura 4).

El valor de $\mathrm{pH}$ promedio más alto se registró en marzo $2017(8.10 \pm 0.12)$ y el valor de $\mathrm{pH}$ más bajo en junio del $2016(6.71 \pm 0.12)$. Si bien es cierto se observa que hay diferencias entre los datos $(\mathrm{p}<0.05)$, en las comparaciones entre los meses, el pH bajó de abril a mayo del 2016, se mantiene constante hasta septiembre (no hubo variaciones significativas), y de allí sube esta variable para el mes de octubre, tomando ligeras variaciones (Figura 5).

Para el oxígeno, se encontraron diferencias significativas $(\mathrm{p}<0.05)$. En las comparaciones entre

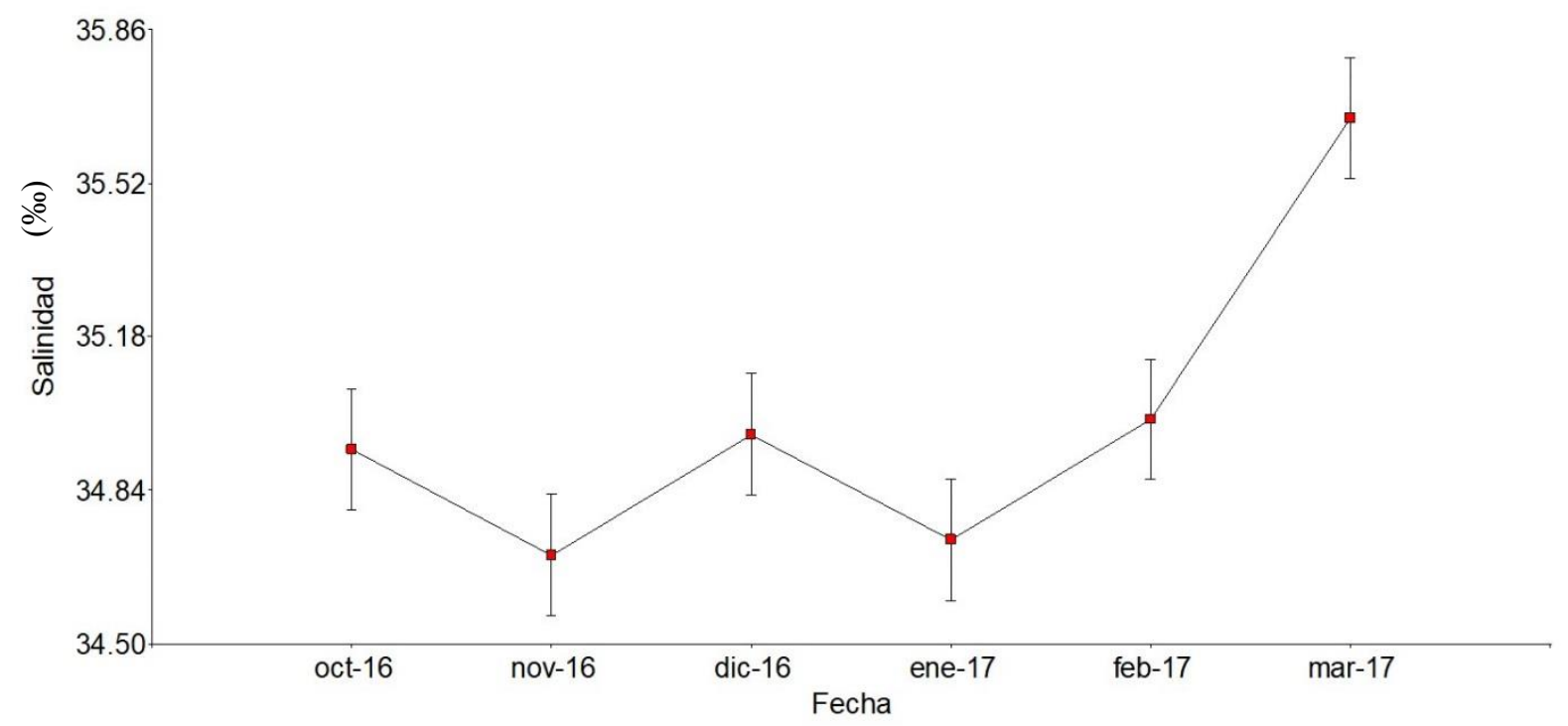

Figura 4. Variación temporal en valores medios $( \pm \mathrm{EE})$ de la salinidad del agua durante el periodo de estudio. 


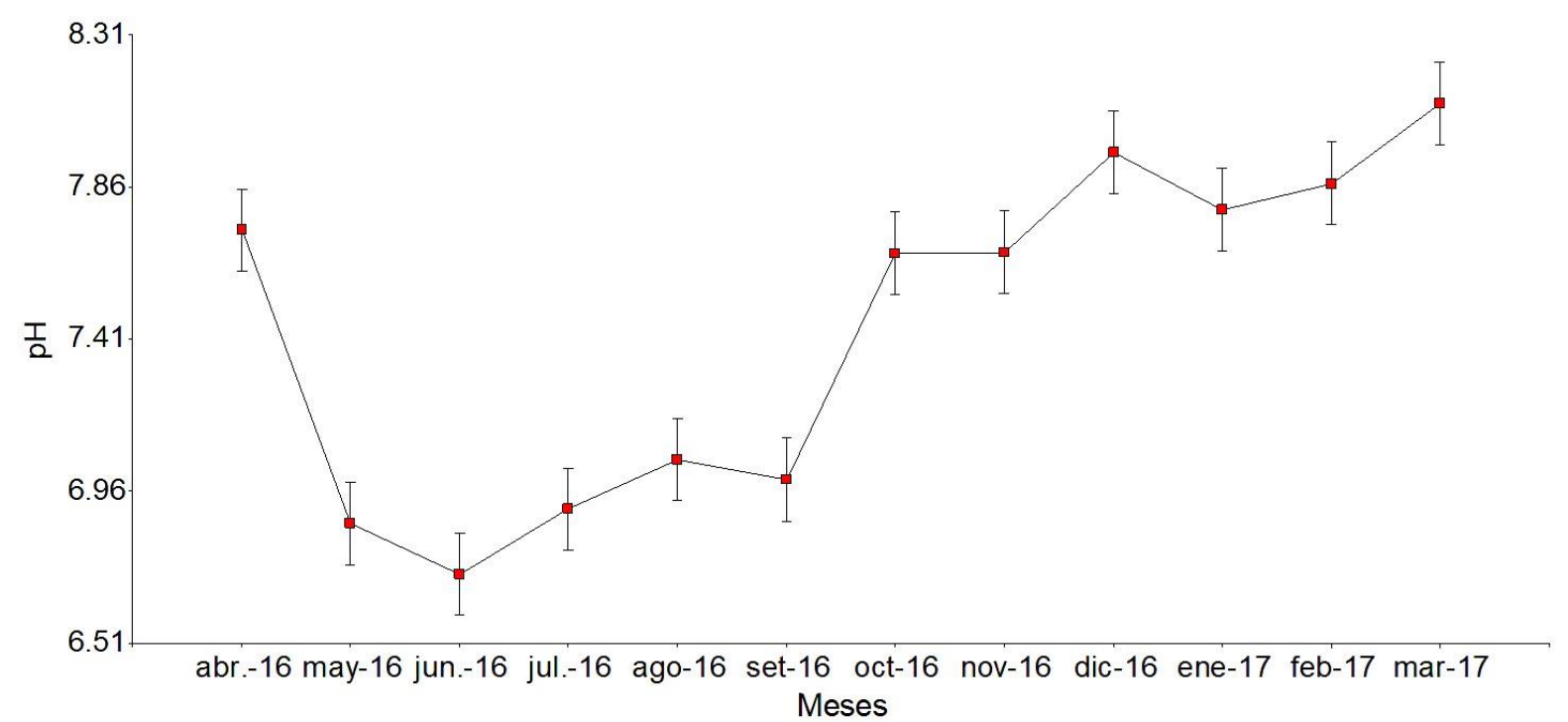

Figura 5. Variación temporal en valores medios $( \pm \mathrm{EE})$ de $\mathrm{pH}$ durante el periodo de estudio.

meses con el LSD de Fisher, indicó que no hay diferencias entre los meses de octubre y noviembre del 2016, aumenta en diciembre, baja entre los meses de enero y febrero de 2017, para finalmente subir en marzo del mismo año. Los picos de oxígeno fueron para diciembre de $2016(9.30 \pm 0.58)$ y marzo del 2017 (8.73 \pm 0.58 ) (Figura 6).

\section{Abundancia de fitoplancton}

Raphidophyceae fue la dominante en número (73.72\%), seguido de dinoflagelados y nanoflagelados $(<20 \mu \mathrm{m})$ con 11.75 y $11.53 \%$ respectivamente. La clase Raphidophyceae dominada por la especie Heterosigma akashiwo presentó abundancias muy altas en otoño (junio 2016) y verano (marzo 2017) y las más bajas en primavera. La clase Dictyochophyceae dominada por la especie Dictyocha fibula resultó con abundancias muy altas en verano (Figura 7).

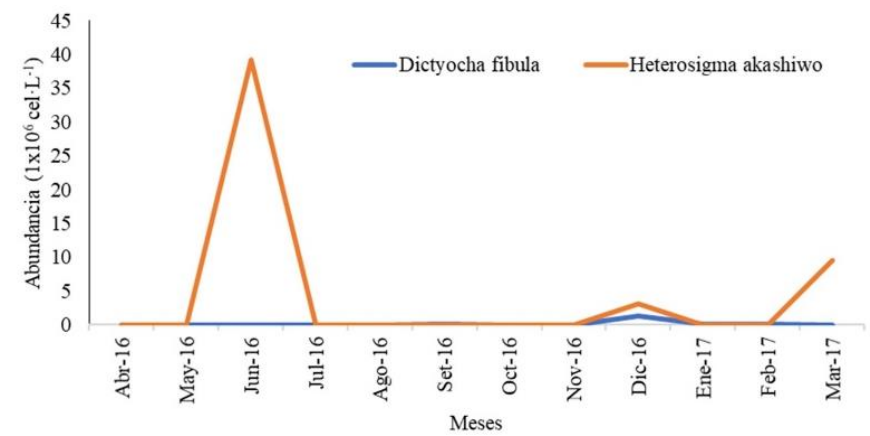

Figura 7. Variación temporal en valores medios de Dictyochophyceae y Raphidophyceae durante el periodo de estudio.

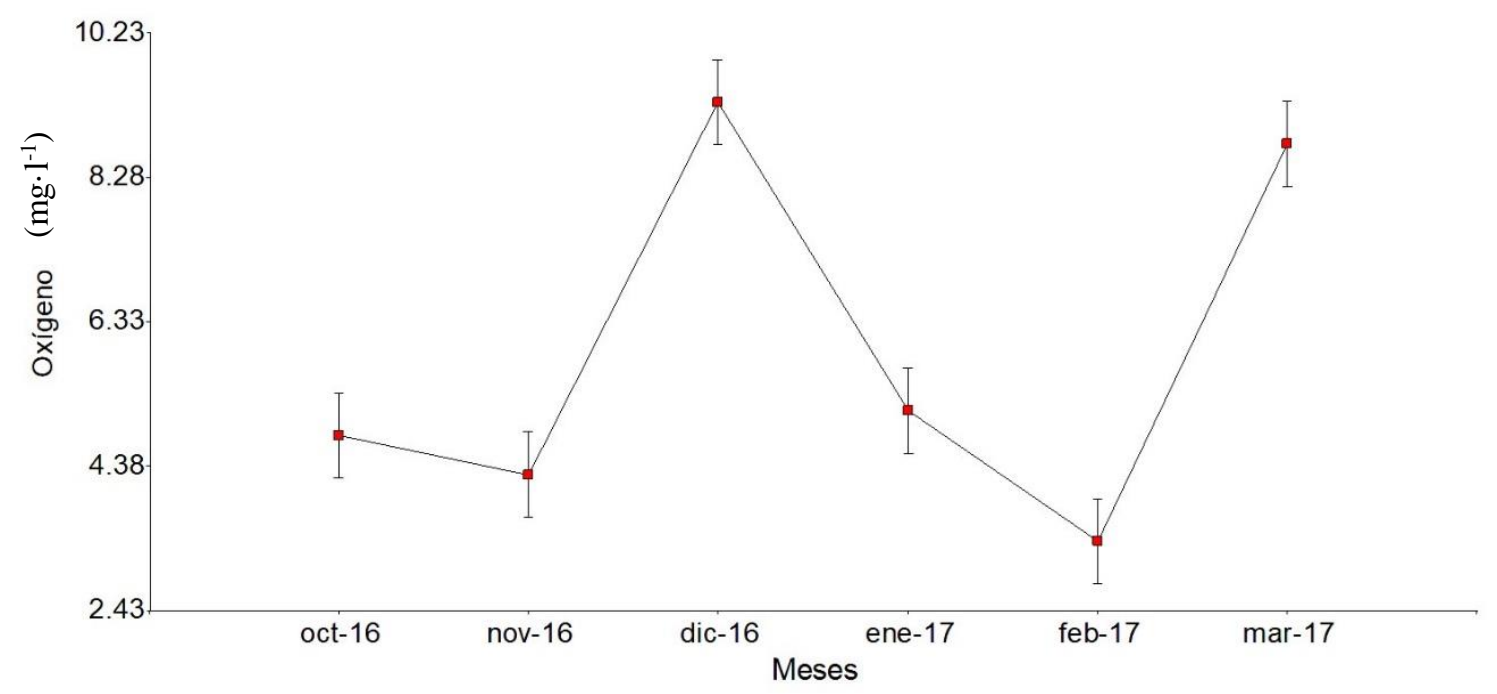

Figura 6. Variación temporal en valores medios ( $\pm \mathrm{EE})$ de oxígeno disuelto durante el periodo de estudio. 
Los dinoflagelados presentaron una alta abundancia en la época de verano y las más baja en invierno, esta alta abundancia se debió principalmente a las especies: Prorocentrum gracile en junio de 2016, $P$. minimum en diciembre de 2016, $P$. balticum en febrero de 2017 y Scripsiella acuminata en marzo de 2017 (Figura 8).

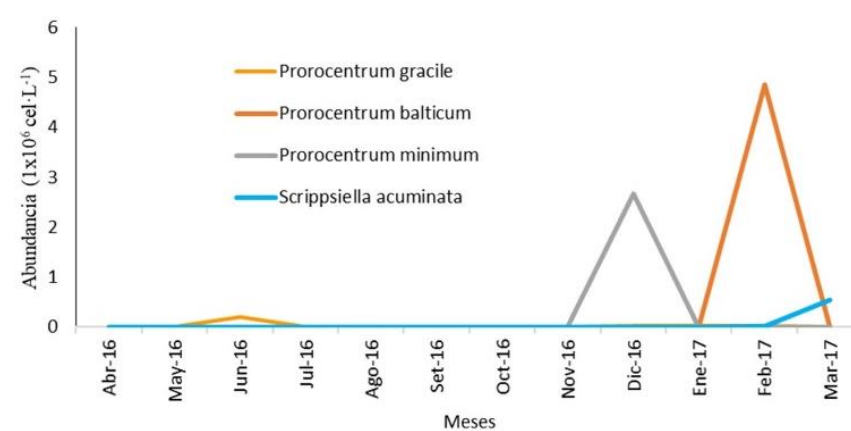

Figura 8. Variación temporal en valores medios de Miozoa durante el periodo de estudio.

La distribución temporal del fitoplancton mostró mayores abundancias de diatomeas en verano y otoño y las más bajas en invierno. Thalassionema nitzschioides fue abundante en mayo 2016 y enero 2017, Nitzschia sp.1 en abril de 2016, Pleurosigma sp. en agosto de 2016 y Skeletonema costatum y Nitzschia longissima en el verano de 2017 (Figura 9).

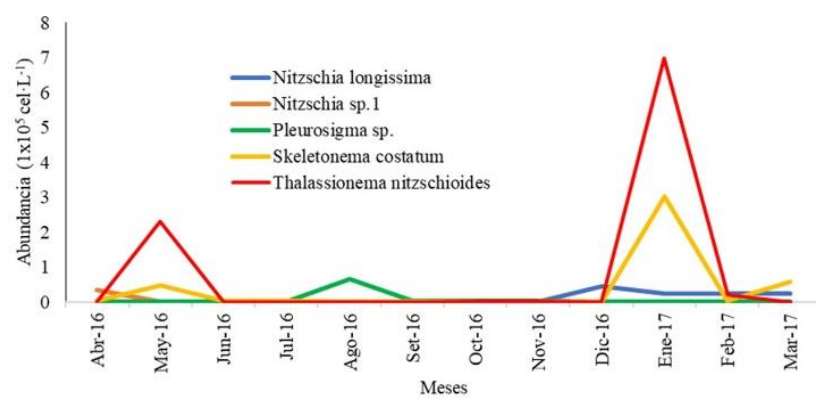

Figura 9. Variación temporal en valores medios de Bacillariophyta durante el periodo de estudio.

Relación entre las variables fisicoquímica y la abundancia de fitoplancton

Los resultados de la correlación de Pearson entre las variables fisicoquímicas y los grupos del fitoplancton evidencian que la variable temperatura correlacionó con los dinoflagelados $(\mathrm{p}=0.00)$ y las variables salinidad y oxígeno disuelto correlacionaron significativamente con Raphidophyceae $(\mathrm{p}=0.01 \mathrm{y} \mathrm{p}=$ 0.00 respectivamente), las variables temperatura y salinidad correlacionaron significativamente con la división Euglenophyta $(p=0.02$ y $p=0)$ y finalmente la variables temperatura y $\mathrm{pH}$ correlacionaron significativamente con los nanoflagelados $(<20 \mu \mathrm{m})(\mathrm{p}$ $=0.01, \mathrm{p}=0.04)($ Tabla 2).
Floraciones Algales

En toda el área de estudio se registraron floraciones algales potencialmente tóxicas/nocivas con valores mayores a $100 \times 10^{3} \mathrm{cel} \cdot 1^{-1}$ originadas por las especies, Thalassionema nitzschioides, Skeletonema costatum, Akashiwo sanguinea, Prorocentrum gracile, $P$. balticum, $P$. minimum, Scrippsiella acuminata, Dictyocha fibula, Heterosigma akashiwo, Leucocryptos marina y nanoflagelados $(<20 \mu \mathrm{m})$, dando una coloración particular al agua de mar, como se muestra en la Tabla 3. Las FAs tuvieron un tiempo de duración de varios días a excepción de $A$. sanguinea y $H$. akashiwo que tuvieron una permanencia de alrededor de un mes (comunicación personal con los pescadores artesanales de la zona).

Tabla 2. Correlaciones de Pearson entre las variables fisicoquímicas y la comunidad fitoplanctónica.

\begin{tabular}{|c|c|c|c|}
\hline División & Variable & 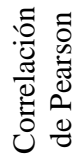 & $\begin{array}{l}\frac{0}{J} \\
\frac{\pi}{7} \\
1 \\
2\end{array}$ \\
\hline Miozoa & Temperatura & 0.59 & 0.00 \\
\hline Raphidophyceae & Salinidad & 0.9 & 0.01 \\
\hline Raphidophyceae & Oxígeno & 0.85 & 0.00 \\
\hline Euglenophyta & Temperatura & 0.67 & 0.02 \\
\hline Euglenophyta & Salinidad & 0.95 & 0.00 \\
\hline Nanoflagelados $(<20 \mu \mathrm{m})$ & Temperatura & 0.72 & 0.01 \\
\hline Nanoflagelados $(<20 \mu \mathrm{m})$ & $\mathrm{pH}$ & 0.58 & 0.04 \\
\hline
\end{tabular}

\section{Discusión}

Ochoa (1988) describe para las áreas neríticas a lo largo del litoral peruano bajas temperaturas del agua de mar: 13 a $17{ }^{\circ} \mathrm{C}$ en invierno y 17 a $23{ }^{\circ} \mathrm{C}$ en verano; mostrando alta concordancia con las TSM en nuestra zona de estudio. Para el periodo de estudio se evidenció valores de salinidad entre 34.8 y $35.9 \%$, encontrando los valores más altos de salinidad y temperatura en verano (febrero y marzo de 2017), lo que indicaría el ingreso de aguas más cálidas y salinas a las áreas neríticas, producida posiblemente por el fenómeno climatológico El Niño. Salvá (2017) registra para el Pacífico Ecuatorial Oriental, un calentamiento anómalo a partir de enero y su intensificación en febrero y marzo 2017, llegando la anomalía hasta $2.5^{\circ} \mathrm{C}$.

Muestreos más intensivos a lo largo de un año en la playa de Pescadores Artesanales de Chorrillos dieron como resultados una alta riqueza de taxa (120), con 71 especies (59.2\%) del phylum Bacillariophyta y 43 especies (35.6\%) del phylum Miozoa comparado con el estudio realizado por Arellano et al. (2006) durante el invierno, en donde se registró para la misma zona solo 39 especies, siendo las más dominantes Skeletonema costatum y Thalassiosira rotula.

Las variables fisicoquímicas y las comunidades fitoplanctónicas no fueron significativamente diferentes entre los tres sitios de muestreo, pero 
mostraron variaciones temporales significativas entre los meses $(\mathrm{p}<0.05)$. La distribución temporal del fitoplancton evidenció que $H$. akashiwo dominó entre otoño y verano, dinoflagelados como $P$. minimum, $P$. balticum, $S$. acuminata, etc. presentaron alta abundancia en la época de verano y las más baja en invierno y algunas especies de diatomeas como Skeletonema costatum, Nitzschia longissima, Pleurosigma sp. dominaron en verano y Thalassionema nitzschioides y Nitzschia sp.1 en otoño. Nuestros resultados son similares a los reportados por Ochoa et al. (2010), quienes correlacionaron los cambios de la periodicidad anual (es decir, la temperatura superficial del mar) con las proporciones de taxones de fitoplancton, encontrando que las diatomeas (por ejemplo, Chaetoceros sp., Actinocyclus octonarius y Skeletonema costatum) dominaron solo durante los períodos más fríos $\left(13-16{ }^{\circ} \mathrm{C}\right)$ cuando el afloramiento es más fuerte, mientras que los dinoflagelados dominan en los períodos más cálidos donde la intrusión de aguas en alta mar aumenta la proporción de fitoplancton de etapas posteriores dentro de la bahía (por ejemplo, dinoflagelados, flagelados y silicoflagelados).

En este estudio se evidenció una correlación positiva en la abundancia de dinoflagelados $(p=0.00)$, euglenofitas $(\mathrm{p}=0.02)$ y nanoflagelados $(<20 \mu \mathrm{m})(\mathrm{p}$ $=0.01)$ con la temperatura. La temperatura es un parámetro clave que afecta directamente las tasas fisiológicas de la biota marina a múltiples escalas, por ejemplo, reacciones enzimáticas, respiración, tamaño corporal, tiempo de generación, interacciones ecológicas, metabolismo comunitario, etc (Peters, 1983).

Raphidophyceae $\left(6.62 \times 10^{6} \mathrm{cel} \cdot \mathrm{l}^{-1}\right)$ correlacionó positivamente con las variables salinidad $(\mathrm{p}=0.01)$ y oxígeno disuelto $(\mathrm{p}=0.03)$. La salinidad co-varía estrechamente con la temperatura y también tiene importantes implicaciones en la fisiología del plancton, afectando la germinación de las etapas de reposo, en las tasas de crecimiento y desarrollo de floraciones en aguas costeras (McQuoid, 2005; Shikata et al., 2008). El oxígeno disuelto es uno de los parámetros de calidad del agua más importantes para la salud del fitoplancton porque tiene influencia en una serie de procesos biogeoquímicos como la respiración y el metabolismo que afectan su vida (Iriarte et al., 2015).

Steidinger (1975) menciona dos características inseparables en todas las floraciones algales; el primero, el incremento del tamaño de la población, y el segundo la disponibilidad de una adecuada salinidad, temperatura y factores de crecimiento como los nutrientes.

En abril de 2016 se presentó una FA de $A$. sanguinea, cuya abundancia $1.74 \times 10^{5} \mathrm{cel} \cdot 1^{-1}$ podría indicar el término de la floración (parches coloreados). Esta floración algal asociado a TSM de $21{ }^{\circ} \mathrm{C}$ y concentraciones de oxígeno disuelto de $7.2 \mathrm{mg} \cdot \mathrm{l}^{-1}$ coincidió con una FA de la misma especie en la bahía
Miraflores, playa Los Delfines, playa Las Cascadas y frente a la Isla San Lorenzo (IMARPE, 2016), también se reportaron FAs de $A$. sanguinea $\left(12 \times 10^{6} \mathrm{cel} \cdot 1^{-1}\right)$, en Bayovar, Matacaballo y Vichayo originando varazones de organismos marinos (30 al 90\% de mortalidad del recurso Argopecten purpuratus) (IMARPE, 2007).

En diciembre de 2016 se presentó en toda el área de estudio una coloración rojiza intensa ocasionada por $H$. akashiwo $\left(4.86 \times 10^{6} \mathrm{cel} \cdot 1^{-1}\right), P$. minimum $\left(4.04 \times 10^{6}\right.$ cel. $\left.1^{-1}\right)$ y $D$. fibula $\left(2.2 \times 10^{6} \mathrm{cel} \cdot 1^{-1}\right)$ propiciando altos valores de oxígeno $\left(10.8 \mathrm{ml} \cdot \mathrm{1}^{-1}\right)$ y $\mathrm{pH}(8.3)$. Las FAs de $P$. minimum se han presentado en una variedad de ambientes costeros (Japón, Francia, Noruega, Países Bajos, Nueva York, EE. UU.), clones aislados de $P$. minimum en la costa mediterránea de Francia y los mariscos expuestos a las floraciones de la zona, han demostrado que contienen un componente neurotóxico que mata a ratones (Heila et. al., 2005). Para las aguas costeras peruanas la toxicidad de $P$. minimum se encuentra aún en discusión. Ruiz (2013) realizó el aislamiento, identificación, clonación y análisis filogenético de $P$. minimum. En sus resultados no logró obtener la toxina DSP (Toxina Diarreica de Moluscos) del cultivo de la microalga, pero sí la purificación del cultivo de $P$. minimum. No se ha constatado para nuestra zona marino-costera que floraciones de $D$. fibula hayan producido efectos nocivos, como el caso de Dictyocha speculum especie que erosiona físicamente e irrita las agallas de los peces (Reguera, 2002).

Las FAs en febrero 2017 de P. balticum $\left(8 \times 10^{6}\right.$ cel $\left.\cdot 1^{-1}\right)$ y Leucocryptos marina $\left(3.8 \times 10^{6} \mathrm{cel} \cdot \mathrm{l}^{-1}\right)$ están relacionadas con altos valores de TSM $\left(22.6{ }^{\circ} \mathrm{C}\right), \mathrm{pH}$ (7.98) y salinidad (35.2). En marzo nuevamente $H$. akashiwo $\left(12 \times 10^{6} \mathrm{cel} \cdot \mathrm{l}^{-1}\right)$ propició altos valores de oxígeno (10 mg. $\left.1^{-1}\right)$, salinidad (35.9\%) y pH (8.32), esta FA tuvo una duración de alrededor de un mes. Vargo (2009) menciona que los parámetros fisicoquímicos tales como temperatura y la salinidad pueden determinar la distribución o la ocurrencia de floraciones algales junto con la disponibilidad de nutrientes que regulan la tasa de crecimiento, la biomasa y la duración de la floración.

Durante todo el periodo de estudio los organismos nanoplanctónicos reflejan floraciones algales en curso, cuyas concentraciones variaron entre $66 \times 10^{3} \mathrm{cel} \cdot \mathrm{l}^{-1} \mathrm{y}$ $3.66 \times 10^{6} \mathrm{cel} \cdot 1^{-1}$. Si comparamos estas densidades con áreas fuertemente eutrofizadas donde fitoflagelados nanoplanctónicos alcanzaron concentraciones celulares mayores a $10^{6} \mathrm{cel} \cdot \mathrm{l}^{-1}$ en el área costera del Callao (Salbatier, 2008), se evidencia procesos de eutrofización existentes, ocasionados por las actividades antropogénicas del muelle, la cual se caracteriza por presentar focos de contaminación, vinculados a aguas servidas, desechos orgánicos de las embarcaciones artesanales, etc. Lam \& Ho (1989) encontraron una correlación positiva entre el número de mareas rojas por año y el incremento de la población en 
Hong Kong. Estas floraciones se dieron principalmente por Karenia mikimotoi, Gonyaulax polygramma, Noctiluca scintillans y Prorocentrum minimum. En series de tiempo de varios años Hallegraeff (1993) muestra correlaciones positivas entre la frecuencia de mareas rojas y los desechos vertidos por la actividad humana. Smayda (1990) afirmó que estas floraciones (a menudo tóxicas) han alcanzado proporciones de epidemia por el incremento de nutrientes. Los pescadores de la zona manifiestan que las "mareas rojas" en la playa de Pescadores Artesanales de Chorrillos han sido más frecuentes en aparición y duración en estos últimos años, lo que no ha impedido que sigan con sus actividades en la pesca artesanal. La existencia de especies de fitoplancton potencialmente tóxico/nocivo sugiere un consumo cuidadoso de los recursos pesqueros en la zona de estudio y un monitoreo frecuente de las floraciones de algas nocivas (FAN).

\section{Conclusiones}

Las especies de fitoplancton fueron dominadas por las diatomeas y dinoflagelados y la abundancia del fitoplancton estuvo dominada por Raphidophyceae $(73.72 \%)$ seguido de dinoflagelados (11.75\%) y nanoflagelados $(<20 \mu \mathrm{m})(11.53 \%)$. La comunidad fitoplanctónica se caracterizó por la presencia de importantes floraciones algales ocasionadas por las especies; Thalassionema nitzschioides, Skeletonema costatum, Akashiwo sanguinea, Heterosigma akashiwo, Prorocentrum gracile, $P$. balticum, $P$. minimum, Scrippsiella acuminata, Dictyocha fibula, Leucocryptos marina y nanoflagelados $(<20 \mu \mathrm{m})$. Las variables físicoquímicas y las comunidades fitoplanctónicas mostraron variaciones temporales significativas entre los meses de estudio. La mayor abundancia de fitoplancton se observó en verano y otoño. Se encontraron correlaciones significativas entre las comunidades del fitoplancton y las variables físicoquímicas, lo que pudo determinar que el oxígeno disuelto, temperatura, salinidad y $\mathrm{pH}$ favorecieron la presencia de las floraciones algales, sumado a ello los fuertes procesos de eutrofización existentes en el área. La existencia de especies de fitoplancton potencialmente tóxico/nocivo sugiere un consumo cuidadoso de los recursos pesqueros en la playa de Pescadores Artesanales de Chorrillos y un monitoreo frecuente de las floraciones de algas nocivas (FAN).

\section{Literatura citada}

Arellano C., Becerra N., Jara M., La Torre M. I. \& Yucra H. 2006. Fitoplancton de la Playa Los Pescadores, Chorrillos, Lima, Perú, invierno 2005. Biologist (Lima), 4(2): 9-11. URI: http://sisbib.unmsm.edu.pe/BVRevistas/biologist/v04 n 2/pdf/a01v4n2.pdf

Balech E. 1988. Dinoflagelados del Atlántico sudoccidental. Publicaciones especiales instituto español de oceanografía. $\mathrm{N}^{\circ}$ 1: 9-309. Madrid.
Balech E. 1995. The Genus Alexandrium Halim (Dinoflagellata), Sherkin Island Marine Station, Ireland.

Baylón M., Sánchez S., Bárcena V., López J. \& Mamani E. 2015. Primer reporte del dinoflagelado potencialmente tóxico Alexandrium minutum Halim 1960 en el litoral peruano. Revista Peruana de Biología. 22(1): 113-118. DOI: https://doi.org/10.15381/rpb.v22i1.11129.

Booth B.C., Lewin J. \& Norris R.E. 1982. Nanoplankton species predominant in the subarctic Pacific in May and June 1978. Deep Sea Research Part A. Oceanographic Research Papers. 29(2): 185-200.

Bustamante A. \& Borda M.B. 1970. La pesquería en la caleta de Chorrillos. IMARPE (Instituto del Mar del Perú). Informe especial $\mathrm{N}^{\circ} 060 . \quad$ URI: http://biblioimarpe.imarpe.gob.pe:8080/bitstream/handle /123456789/1628/INF\%20ESP.\%2060.pdf?sequence=1.

Cupp E.E. 1943. Marine plankton diatoms of the west coast of North America. Bulletin of the Scripps Institution of Oceanography. 5(1): 1-238. University of California Press, Berkeley and Los Angeles. Permalink. URI: https://escholarship.org/uc/item/922945w8.

Ekwu A.O. \& Sikoki F. 2006. Phytoplankton Diversity in the Cross River Estuary of Nigeria. Journal of Applied Sciences and Environmental Management, 10(1): 89-95. DOI: $\underline{10.10 .4314 / \text { jasem.v10i1.17296. }}$.

Graco M.I., Ledesma J., Flores G. \& Girón M. 2007. Nutrientes, oxígeno y procesos biogeoquímicos en el sistema de surgencias de la corriente de Humboldt frente a Perú. Revista Peruana de Biología. 14(1): 117-128. DOI: https://doi.org/10.15381/rpb.v14i1.2165.

Hallegraeff G.M. 1981. Seasonal study of phytoplankton pigments and species at a coastal station off Sydney: importance of diatoms and the nanoplankton. Marine Biology. 61(2-3): 107-118. DOI: https://doi.org/10.1007/BF00386650.

Hallegraeff G.M. 1983. Scale-bearing and loricate nanoplankton from the East Australian Current. Botanica marina, 26(11): 493-516.

DOI: $\underline{10.1515 / \text { botm.1983.26.11.493 }}$

Hallegraeff G.M \& Jeffrey S. 1984. Tropical phytoplankton species and pigments of continental shelf waters of north and north-west Australia. Marine ecology progress series, 20(1): 59-74. http://www.jstor.org/stable/44634646.

Hallegraeff G.M. 1993. A review of harmful algal blooms and their apparent global increase. Phycologia, 32(2): 79-99. Taylor \& Francis Group. Abingdon, UK. DOI: 10.2216/i0031-8884-32-2-79.1.

Hasle G.R. \& Syvertsen E.E. 1997. Marine diatoms. In: Tomas C.R. (Ed.). 1997. Identifying Marine Phytoplankton, 5-385.

Hernández-Becerril D.U. 1993. Fitoplancton marino en México. Biodiversidad marina y costera de México, 3953.

Heila C.A., Glibert P.M. \& Fan C. 2005. Prorocentrum minimum (Pavillard) Schiller: a review of a harmful algal bloom species of growing worldwide importance. Harmful Algae, 4(3): 449-470. DOI: 10.1016/j.hal.2004.08.003.

Hustedt F. 1930. Bacillariophyta (diatomeae). In: Die Süsswasser-Flora Mitteleuropas, Deutschlands, Österreichs und der Schweiz, heft 10: 1-466. DOI: https://doi.org/10.5962/bhl.title.21607. 
IMARPE (Instituto del Mar del Perú). 2007. Estudio de línea base del ámbito marino de la Bahía de Sechura, $14-28$ enero 2007. Callao, Perú.

IMARPE (Instituto del Mar del Perú). 2016. Reporte de floración algal (marea roja) en playa Carpayo, Los Delfines, Las Cascadas e isla San Lorenzo - marzo 2016. Nota de prensa. Callao, Perú. URI http://www.imarpe.gob.pe/imarpe/archivos/noticia/imar pe_notic_marea_roja_edit.pdf.

Iriarte A., Villate F., Uriarte I., Alberdi L. \& Intxausti L. 2015. Dissolved oxygen in a temperate estuary: the Influence of hydroclimatic factors and eutrophication at seasonal and inter-annual time scales. Estuar Coasts, 38(3): 1000-1015.

Kameya A. 2002. Características ecológicas del Mar Peruano. Primer seminario Virtual de las Ciencias del Mar OANNES. Perú.

URI: http://www.oannes.org.pe/upload/201609221034379691 71575.pdf.

Lam C.W. \& Ho K.C. 1989. Phytoplankton characteristics of Tolo Harbour. Asian Marine Biology, 6: 5-18.

Licea S., Moreno J.L, Santoyo H. \& Figueroa G. 1995. Dinoflageladas del Golfo de California Universidad Autónoma de Baja California Sur (Editor).

Limbu S.M. \& Kyewalyanga M.S. 2015. Spatial and temporal variations in environmental variables in relation to phytoplankton composition and biomass in coral reef areas around Unguja, Zanzibar, Tanzania. SpringerPlus, 4(646): 13-16. DOI: 10.1186/s40064-015-1439-z.

McQuoid M. 2005. Influence of salinity on seasonal germination of resting stages and composition of microplankton on the Swedish west coast. Mar. Ecol. Prog. $\quad$ Ser., 289: 151-163. https://www.jstor.org/stable/24867998

Malone T. 1971a. The relative importance of nannoplankton and netplankton as primary producers in tropical oceanic and neritic phytoplankton communities. Limnology and Oceanography. 16(4): 633-639.

Malone T. 1971b. Diurnal rhythms in netplankton and nannoplankton assimilation ratios. Marine Biology. 10(4): 285-289.

Ochoa L. 1988. Dinoflagelados del mar peruano y su valor como indicadores de masas de agua. Tesis doctoral. Universidad Nacional Mayor de San Marcos. Perú.

Ochoa L. \& Gómez O. 1988. Variación espacio-temporal del fitoplancton frente a Callao, Perú, en 1986. Bol. Inst. Mar Perú. Vol. Extraordinario: 51-57.

Ochoa N., Taylor M. H., Purca S. \& Ramos E. 2010. Intraand interannual variability of nearshore phytoplankton biovolume and community changes in the northern Humboldt Current system. Journal of Plankton Research, 32(6): 843-855.

Peters R.H. 1983. The ecological implications of body size. Cambridge University Press, Cambridge.

Reguera B. 2002. Estabelecimiento de un programa de seguimiento de microalgas toxicas. En: Sar E.A., Ferrario M.E. \& Reguera B. (eds) Floraciones algales nocivas en el Cono Sur Americano. 21-54. Instituto Español de Oceanografía.

Rojas de Mendiola B. 1979. Red tide along the Peruvian coast. Toxic dinoflagellate blooms, 1: 183-190.
Round F., Crawford R. \& Mann D. 1990. Diatoms: biology and morphology of the genera. Cambridge University Press.

Ruiz C.M. 2013. Aislamiento, identificación, clonación y análisis filogenético de la microalga Prorocentrum minimum (Pavillard) J. Schiller 1933 aislada de la Bahía del Callao-Perú. Tesis de maestría. Universidad Nacional Mayor de San Marcos. Perú.

Salbatier M., Osorio S., Fajardo W., Roncal F. \& Olivares V. 2008. Caracterización fitoplanctónica del área Costera del Callao-Perú, afectada por aguas residuales. The Biologist. 6(2): 135-145.

Salvá A. 2017. Calentamiento anómalo costero 2017. Boletin ASP Calentamiento Anómalo Costero 19: 1-17.

Sánchez S. \& Delgado E. 1996. Mareas rojas en el área del Callao (12 $\left.{ }^{\circ} \mathrm{S}\right)$ 1980-1995. IMARPE, Informe progresivo 44: $19-37$

Sánchez S., Delgado E., Bances S., Quintana P. \& Huamani A. 2015. Floraciones algales en aguas costeras del mar peruano Paita-Ilo. Instituto del Mar del Perú, 3.

Santander H. \& Ochoa N. 1981. Mareas rojas en el Plancton del Pacífico Oriental. Informe de la UNESCO sobre ciencias del mar 19: 18-23.

Shikata T.S., Nagasoe S., Matsubara T., Yoshisawa S., Yamasaki Y., Shimasaki Y., Oshima Y., Jenkinson I.R \& Honjo T. 2008. Factors influencing the initiation of blooms of the raphidophyte Heterosigma akashiwo and the diatom Skeletonema costatum in a port in Japan. Limnol. Oceanogr., 53(6): 2503-2518. DOI: https://doi.org/10.4319/1o.2008.53.6.2503.

Smayda T. 1990. Novel and nuisance phytoplankton blooms in the sea evidence for a global epidemic. In: Toxic Marine Phytoplankton: Proceedings of the Fourth International Conference on Toxic Marine Phytoplankton, Held June 26-30 in Lund, Sweden. 29-40. Elsevier Science Publishing.

Steidinger K. 1975. Basic factor influencing red tides; Contribution $\mathrm{N}^{\circ} 246$. In: LoCicero V.R. (Editor) Proceedings of The First International Conference on Toxic Dinoflagellate Blooms, November 1974, Boston, Massachusetts. 153-162. Florida Department of Natural Resources Marine Research Laboratory.

Tillmann U., Sánchez-Ramírez S., Krock B. \& BernalesJiménez A. 2017. A bloom of Azadinium polongum in coastal waters off Peru. Revista de Biología Marina y Oceanografía, 52(3), 591-610.

Throndsen J. 1978. Preservation and storage. In: Sournia A. (Editor) Phytoplankton manual. 69-74. United Nations Educational, Scientific and Cultural Organization.

Throndsen J. 1979. The significance of ultraplankton in marine primary production. Acta Bot. Fennica, 110: 5356.

Throndsen J. 1993. The Planktonic Marine Flagellates In: Tomas C.R. (ed) Identifying marine phytoplankton. Academic Press.

Utermöhl H. 1958. Zur Vervollkommnung der quantitativen Phytoplankton-Methodik. Mitt. Int. Verein. Theor. Angew. Limnol., 9: 1-38.

Vargo G.A. 2009. A brief summary of the physiology and ecology of Karenia brevis Davis (G. Hansen and Moestrup comb. nov.) red tides on the West Florida Shelf and of hypotheses posed for their initiation, growth, maintenance, and termination. Harmful Algae, 8 (4): 573584. 


\section{Apéndice}

Tabla 3. Lista de floraciones algales (se señala máxima abundancia cel $\cdot l^{-1}$ entre las tres estaciones de muestreo) en la playa de Pescadores Artesanales de Chorrillos, Lima, Perú, durante el periodo de estudio (en rojo valores $>10^{6}$ )

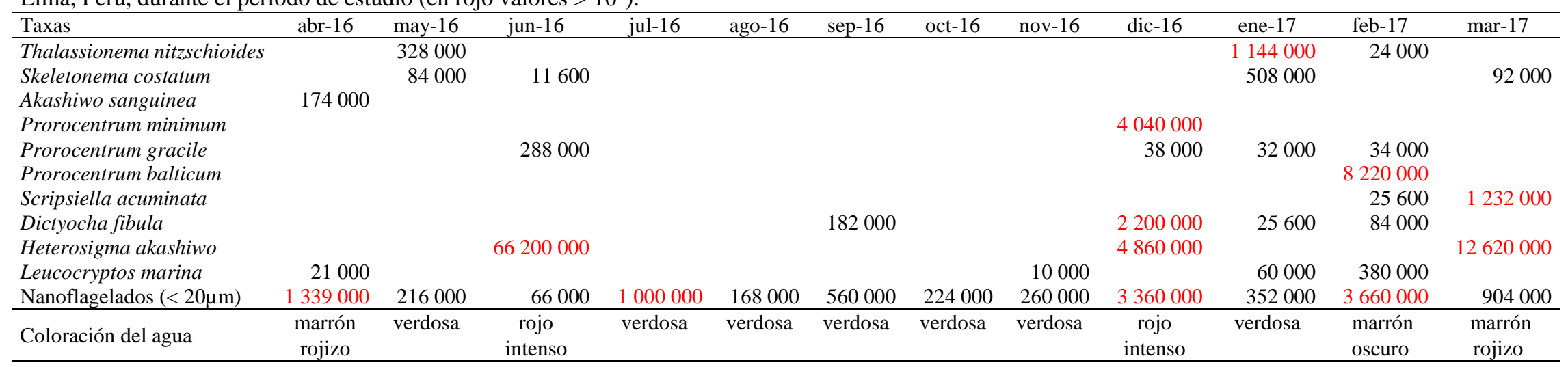

${ }^{1}$ Laboratorio de Ecología Acuática / Facultad de Ciencias Biológicas / Universidad Nacional Mayor de San Marcos (UNMSM). Lima / Perú.

2 mbaylonc@unmsm.edu.pe.

3 Universidad San Ignacio de Loyola. Facultad de Ingeniería. orlandoad@gmail.com.

alejandrolbartra@gmail.com.

5 a.norabuena@hotmail.com.

${ }^{6}$ Instituto de Ciencias del Mar y Limnología, Universidad Nacional Autónoma de México. dhernand@ @marl.unam.mx. 